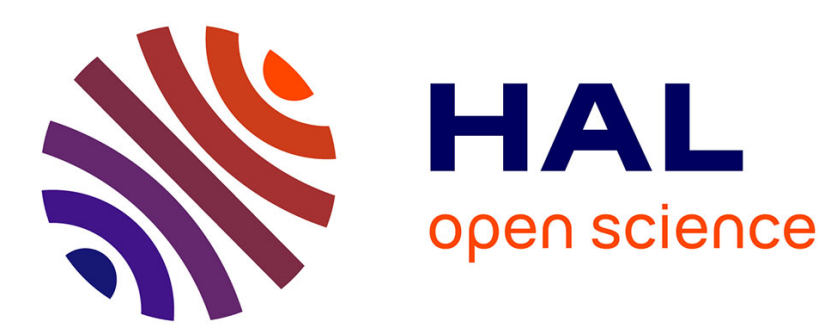

\title{
EFFECT OF SHORT RANGE ORDER ON THE ELECTRONIC STRUCTURE OF DISORDERED TRANSITION ALLOYS
}

\author{
M. Cyrot, F. Cyrot-Lackmann, M. Desjonquères, J. Gaspard
}

\section{- To cite this version:}

M. Cyrot, F. Cyrot-Lackmann, M. Desjonquères, J. Gaspard. EFFECT OF SHORT RANGE ORDER ON THE ELECTRONIC STRUCTURE OF DISORDERED TRANSITION ALLOYS. Journal de Physique Colloques, 1977, 38 (C7), pp.C7-285-C7-288. 10.1051/jphyscol:1977757 . jpa-00217260

HAL Id: jpa-00217260

https://hal.science/jpa-00217260

Submitted on 1 Jan 1977

HAL is a multi-disciplinary open access archive for the deposit and dissemination of scientific research documents, whether they are published or not. The documents may come from teaching and research institutions in France or abroad, or from public or private research centers.
L'archive ouverte pluridisciplinaire HAL, est destinée au dépôt et à la diffusion de documents scientifiques de niveau recherche, publiés ou non, émanant des établissements d'enseignement et de recherche français ou étrangers, des laboratoires publics ou privés. 


\title{
EFFECT OF SHORT RANGE ORDER ON THE ELECTRONIC STRUCTURE OF DISORDERED TRANSITION ALLOYS
}

\author{
M. CYROT $\left({ }^{*}\right)$, F. CYROT-LACKMANN $\left({ }^{* *}\right)$, M. C. DESJONQUÈRES $(* *)$ and J. P. GASPARD (***) \\ C.N.R.S., B.P. 166, 38042 Grenoble Cedex, France
}

\begin{abstract}
Résumé. - Nous présentons le premier calcul a priori d'ordre local dans les métaux de transition. Il est basé sur le calcul des densités d'états locales en fonction de l'environnement. La statistique est introduite dans le modèle de l'atome entouré. Des résultats sont présentés sur l'exemple d'alliages FeNi paramagnétiques.
\end{abstract}

Abstract. - We present the first a priori calculation of the short range order in transitional alloys. This is based on the calculation of local densities of states as a function of environment. The statistics is introduced in the surrounded atom model. Results are presented on the example of FeNi paramagnetic alloys.

Local densities of states, as a function of environment, in disordered transition alloys are particularly important to know in a number of problems. For instance, as we will show in this paper, calculation of short range order requires these quantities in a precise way. This leads also to the study of order disorder transition in these alloys. Other examples include the study of the possible magnetic state of an atom as a function of environment and our theory applies directly to test the Jaccarino-Walker model.

To investigate the electronic structure of disordered transition alloys, the Coherent Potential Approximation [1] (C.P.A.) is the usual starting point. However this approximation is of mean field type and effects of environment can be studied only in a BethePeierls approach. This has been developed [2] but it appeared that difficulties in numerical computations are encountered. Falicov and Yndurain [3] then proposed to explicitly diagonalize a real cluster of atoms and simulate the environment of the cluster by connecting each atom at the surface to a Bethe lattice of the same coordination number. However the lack of interconnections between the different Bethe lattices makes the density of states very similar to a finite cluster density of states. Two of us [4] recently proposed to insert a real cluster in an effective medium in order to obtain reliable results. They choose as the effective medium, the C.P.A. 'one. The agreement with exact results [5] obtained through computer simulation. is very good. Here, we propose an a priori

(*) Laboratoire de Magnétisme.

(**) Groupe des Transitions de Phases.

(***) Groupe des Transitions de Phases et Institut de Physique, Université de Liège (Belgique). calculation of short range order in transition alloys. We first extend the method [4] of calculating the local density of states to take into account the difference between the bandwidths of the two components of the alloy i.e. the off diagonal disorder. Then we introduce self consistency in order to realistically include charge transfer effects. As we will show this is particularly important for short range order. We show how to calculate the short range order Cowley parameter [6] using the surrounded atom model [7] and we present some local densities of states of NiFe alloys.

We start from the usual tight binding model hamiltonian for alloys

$$
\mathscr{H}=\sum \varepsilon_{i} n_{i}+\sum_{i j} t_{i j} C_{i}^{+} C_{j}
$$

$\varepsilon_{i}$ is the effective atomic level on site $i$. This is a random parameter which can take two values $\varepsilon_{A}$ or $\varepsilon_{B}$ for a binary alloy AB. $t_{i j}$ is the hopping integral which can take three values according to the occupancies of sites $i$ and $j: t_{\mathrm{AA}}, t_{\mathrm{BB}}, t_{\mathrm{AB}}$. We consider one central atom and its first shell of $Z$ nearest neighbours. We insert it in an infinite medium made of effective atoms and effective hopping integrals. For the medium, we choose the one calculated by Shiba in its generalisation of C.P.A. to off diagonal disorder [8]. We thus calculate the Green's function on the central atom surrounded by $N$ atoms $\mathrm{A}$ and $Z-N$ atom $\mathrm{B}$. We use the same technique as in the Bethe Peierls [2] and Miwa [9] approximations. The result is

$$
\begin{aligned}
& G_{0}(z)=-\frac{1}{W_{0}} \times \\
& \times \frac{1}{\mathcal{L}_{0}^{-1}-t^{2}\left|\left(\sum_{n} \frac{1}{\mathcal{L}_{n}^{-1}-(S-T)}\right)^{-1}-T\right|^{-1}}
\end{aligned}
$$


where $S$ and $T$ represent walks in the effective medium starting from a nearest neighbour of the central atom and coming back to the same $(S)$ or to a different $(T)$ nearest neighbour, without reaching the first shell as an intermediate step, $W_{0}=W_{\mathrm{A}}$ or $W_{\mathrm{B}}$ according to the nature of the central site and $\varepsilon_{i}$ is the renormalized Shiba locator. The summation over $n$ extends over the first shell of neighbours. $S$ and $T$ are given by the set of equations :

$$
T=\frac{t^{2}\left(z-\Sigma-G^{-1}\right)+Z t^{2} G^{-1}-\frac{Z^{2} t^{4}}{z-\Sigma-G^{-1}}}{Z^{2} t^{2}-(z-\Sigma)\left(z-\Sigma-G^{-1}\right)}
$$

$$
S-T=-Z T+z-\Sigma-\frac{Z t^{2}}{z-\Sigma-G^{-1}} .
$$

Equations (1), (2) and (3) permit us to calculate the local density of states as a function of environment by taking the imaginary part of the Green's function.

A central problem in any realistic calculation is the input parameters. This is directly linked to charge transfers which are of major importance for the study of local order. The hopping integrals $t_{\mathrm{AA}}$ and $t_{\mathrm{BB}}$ are usually fitted to the bandwidth of pure metals $A$ and $\mathrm{B}$. The effective atomic levels $\varepsilon_{\mathrm{A}}$ and $\varepsilon_{\mathrm{B}}$ are taken as the atomic one's $\varepsilon_{\mathrm{A}}^{0}$ and $\varepsilon_{\mathrm{B}}^{0}$ corrected in a Hartree approximation

$$
\varepsilon_{\mathrm{A}}=\varepsilon_{\mathrm{A}}^{0}+U_{\mathrm{A}} \delta n_{\mathrm{A}}
$$

where $U_{\mathrm{A}}$ is the intra atomic Coulomb interaction and $\delta n_{\mathrm{A}}$ the charge transfer on site $\mathrm{A}$. The result for $\delta=\varepsilon_{\mathrm{A}}-\varepsilon_{\mathrm{B}}$ for $\mathrm{NiFe}$ alloys as a function of concentration is given on figure 1 . The value of $\delta$ is very insensitive to the chosen value of $U$, but the charge

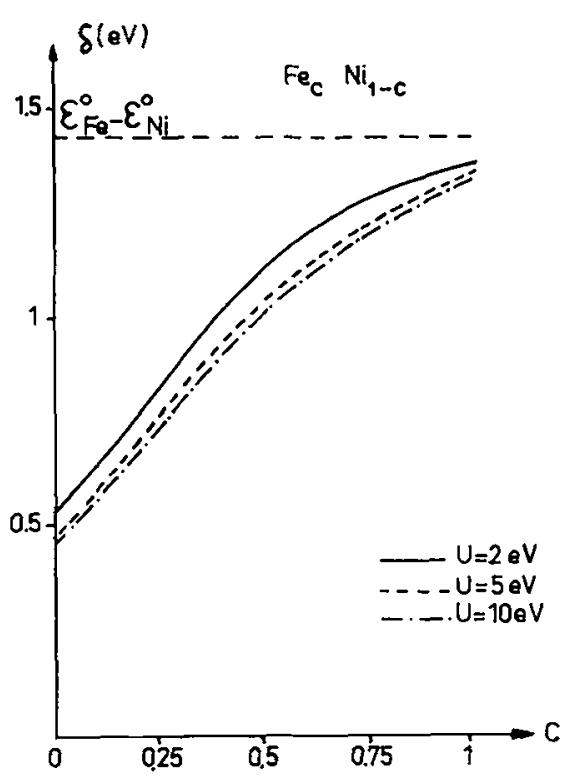

FIG. 1. - Distance between the effective atomic levels in completely random $\mathrm{Fe}_{c} \mathrm{Ni}_{1-c}$ alloys, as a function of the concentration $c$. transfer is very sensitive to it. For these alloys, the electron transfer is from iron to nickel. For low concentrations in iron, the Fermi energy is very close to the Fermi energy of nickel and a large charge transfer appears on iron atoms leading to a strongly reduced value of $\delta$. For a high concentration in iron, the Fermi energy is close to the Fermi energy of iron but this leads to a smaller charge transfer due to the smaller d density of states at the Fermi level in nickel. Thus the value of $\delta$ is not very much renormalised. The difference between the effective atomic levels is thus quite sensitive to the alloy concentration. In the following, calculations have been made with $U_{\mathrm{A}}=U_{\mathrm{B}}=5 \mathrm{eV}$.

To calculate the short range order, we have to introduce the statistics in a manner consistent with our type of approximation. We choose the model of the surrounded atom which is an extension of the quasi chemical model of Guggenheim. This has been extensively used in references [7]. In this model, the energy of the alloy depends on the number $N C_{Z}^{j} m j$ of A atoms surrounded by $j$ atoms B in $C_{z}^{j}$ manners and the number $N C_{Z}^{i} p_{i}$ of $\mathrm{B}$ atoms surrounded by $i$ atoms $\mathrm{A}$ in $C_{Z}^{i}$ manners. The change in energy when alloying is written as

$$
\Delta E=\sum_{j=0}^{Z} C_{Z}^{j} m_{j} U_{j}+\sum_{i=0}^{Z} C_{Z}^{i} p_{i} V_{i}
$$

To make the link with the electronic structure calculations, we define the energy $\varepsilon$ of an atom as the integral up to the Fermi level of $E$ times, the local density of states on this atom i.e.

$$
\varepsilon=\int^{E_{\mathbf{F}}} \operatorname{En}(E) \mathrm{d} E
$$

$U_{j}\left(V_{i}\right)$ is the difference between the energy of an $\mathrm{A}(\mathrm{B})$ atom surrounded by $j \mathrm{~B}(i \mathrm{~A})$ atoms and the energy of an $A(B)$ atom in the pure metal $A(B)$.

The quantities $U_{j}$ and $V_{i}$ are the basic quantities introduced in the model of the surrounded atom. To determine the values of $m_{j}$ and $p_{i}$ which give the probabilities of the different surrounded atoms, we write the partition function as :

$$
\Omega=\sum_{m_{j}, p_{i}} g\left(m_{j}, p_{i}\right) \exp (-\Delta E / k T)
$$

$g$ is the degeneracy of the state of energy $\Delta E$ and is given in references [7]. Following these authors, we approximate the value of $\Omega$ by the largest term in the sum, this determines the values of $m_{j}$ and $p_{i}$ by the set of equations

$$
\frac{\partial \operatorname{Ln} \Omega}{\partial m_{j}}=0 \quad \frac{\partial \operatorname{Ln} \Omega}{\partial p_{i}}=0 .
$$

The calculated value $m_{j}$ is the probability in the alloys 
of having an A atom surrounded by $j$ atoms B. In the completely disordered case, we have

$$
m_{j}=m_{j}^{0}=c c^{Z-j}(1-c)^{j}
$$

where $c$ is the concentration of $\mathrm{A}$ atoms. To define a short range order parameter $\alpha$, we calculate the average number $\bar{j}$ of $\mathrm{B}$ atoms surrounding an $\mathrm{A}$ atom

$$
\bar{j}=\frac{\sum_{j=1}^{Z} j C_{Z}^{j} m_{j}}{\sum_{j=0}^{Z} C_{Z}^{j} m_{j}}
$$

the short range order parameter is

$$
\alpha=1-\frac{\bar{j}}{Z(1-c)}
$$

which is zero for a completely disordered case, positive if the alloy segregates and negative if there is an alternate ordering.

\section{TABLE I}

Parameters of $\mathrm{Ni}$ and $\mathrm{Fe}:$ band filling $Z_{\mathrm{d}}$, bandwidth $W$ and distance between free $3 \mathrm{~d}^{n+1} 4 \mathrm{~s}$ atomic levels.

$\begin{array}{cccc} & Z_{\mathrm{d}} & W(\mathrm{eV}) & \left(\varepsilon_{\mathrm{Fe}}^{0}-\varepsilon_{\mathrm{Ni}}^{0}\right)(\mathrm{eV}) \\ \mathrm{Ni} & 9.4 & 4.36 & 1.4 \\ \mathrm{Fe} & 7 & 5.4 & 1.4\end{array}$

We have done some preliminary calculations along this scheme for FeNi paramagnetic alloys using parameters given in table $\mathrm{I}$. We came to the conclusion that the energy is very sensitive to the chosen parameters. As a consequence, it seems difficult for the moment to derive quantitative predictions of the thermodynamic quantities. However, some quantities such as the short range order parameter are not too sensitive to the input parameters.

Figure 2 gives the density of states of the $\mathrm{Fe}_{50} \mathrm{Ni}_{50}$ completely random alloy compared with the corres-

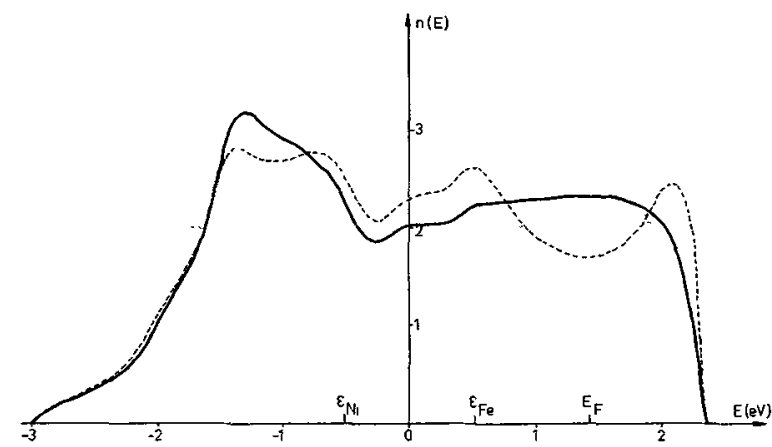

FIG. 2. - Density of states of the $\mathrm{Fe}_{50} \mathrm{Ni}_{50}$ alloy : (-) completely random case $(\alpha=0),(-\ldots)$ partial segregation resulting from a surrounded atom treatment $(\alpha=0.43, T=500 \mathrm{~K})$. ponding curve with partial segregation. The most striking feature is the important decrease of the density of states at the Fermi level. It corresponds to a stabilization of the segregated phase as can be seen in table II. This is related to the different

\section{TABLE II}

Cowley order parameter and enthalpy of formation of $\mathrm{Fe}_{50} \mathrm{Ni}_{50}(\alpha=0.43$ corresponds to $T=500 \mathrm{~K})$

$\begin{array}{ccc}\alpha & 0 & 0.43 \\ \Delta \bar{H}(\mathrm{eV}) & \overline{-11} & -\overline{0} .02\end{array}$

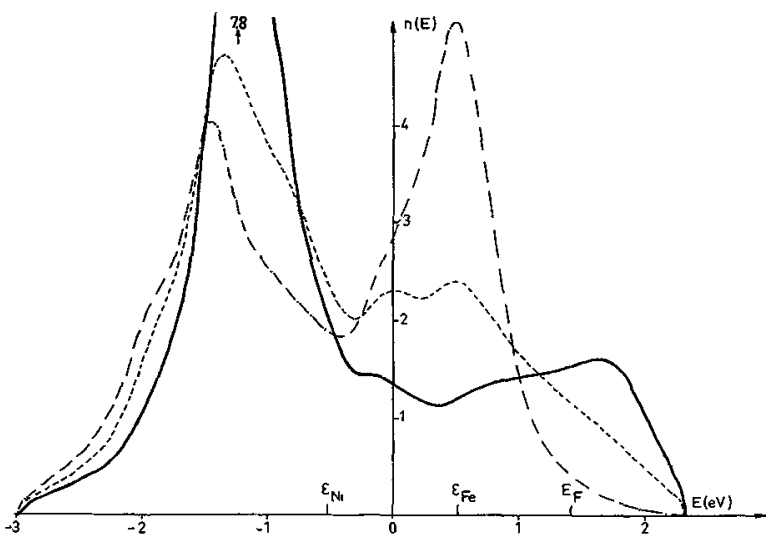

(a)

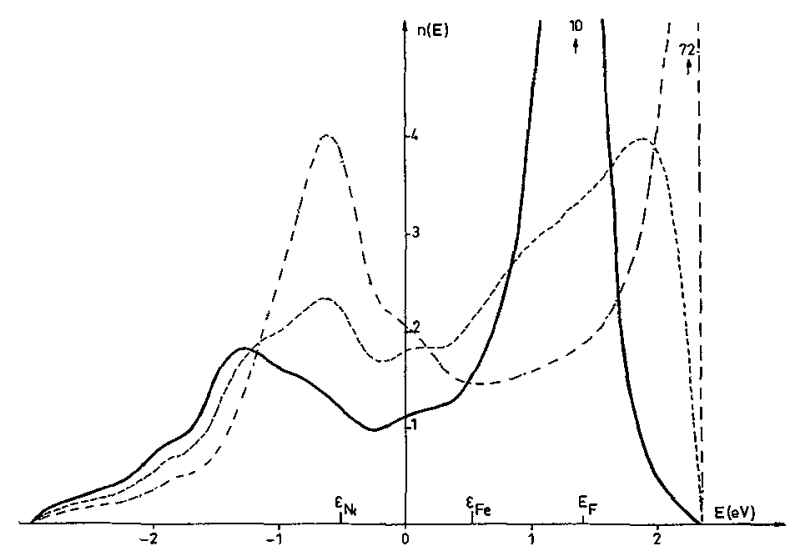

(b)

Fig. 3. - Partial densities of states of a $\mathrm{Fe}_{50} \mathrm{Ni}_{50}$ alloy $(\delta=1.04 \mathrm{eV})$ as a function of environment : $(a)$ on a $\mathrm{Ni}$ atom (一.-.) $\mathrm{Ni}(12 \mathrm{Ni}),(-\ldots.) \mathrm{Ni}(6 \mathrm{Fe}, 6 \mathrm{Ni}),(\longrightarrow) \mathrm{Ni}(12 \mathrm{Fe})$; (b) on a $\mathrm{Fe}$ atom (-...) $\mathrm{Fe}(12 \mathrm{Fe}),(-\ldots) \mathrm{Fe}(6 \mathrm{Ni}, 6 \mathrm{Fe})$, $\rightarrow \mathrm{Fe}(12 \mathrm{Ni})$.

weights of the partial densities of states (figure 3 ). Indeed, the weight of a $\mathrm{Fe}$ (or $\mathrm{Ni}$ ) atom surrounded by $12 \mathrm{Ni}$ (or $\mathrm{Fe}$ ) atoms - configuration which has a high density of states at the Fermi level - is strongly reduced in comparison with the completely random case. On the contrary $\mathrm{Fe}(12 \mathrm{Fe})$ and $\mathrm{Ni}(12 \mathrm{Ni})$ have an increased weight which gives rise to the two 
peaks on each side of the Fermi level. This tendency to segregation is in agreement with the fact that the existence of an ordered fec structure for $\mathrm{Fe}_{50} \mathrm{Ni}_{50}$ seems actually doubtful [10]. Figure 4 gives the beha-

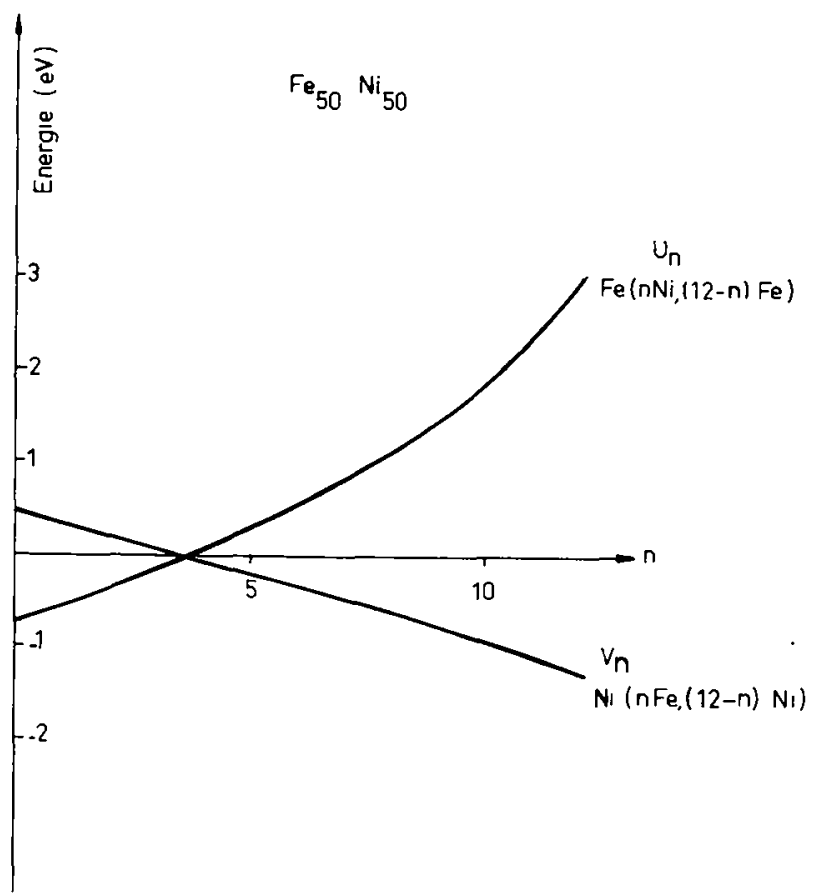

Fig. 4. - Behaviour of $U$ and $V$ for $\mathrm{Fe}_{50} \mathrm{Ni}_{50}$ as a function of $j$ (number of $\mathrm{Ni}$ atoms surrounding a $\mathrm{Fe}$ atom) and $i$ (number of $\mathrm{Fe}$ atoms surrounding a $\mathrm{Ni}$ atom).

viour of $U_{j}$ and $V_{i}$ as a function of $j$ and $i$ for $\mathrm{Fe}_{50} \mathrm{Ni}_{50}$. The energy of a $\mathrm{Ni}$ atom decreases with the number of $\mathrm{Fe}$ atoms nearest neighbours because the electronic states on $\mathrm{Ni}$ increases in energy leading to a less filled band (see figure 3 ). The reverse occurs for a $\mathrm{Fe}$ atom.
The variations of $U_{j}$ and $V_{i}$ are rather large for $\mathrm{Fe}_{50} \mathrm{Ni}_{50}$. Preliminary calculations for some other alloys have shown similar trends. Let us notice that these variations are much smaller for alloys between two neighbouring elements in the periodic table.

We have also performed some calculations for other values of concentration. Results for $\mathrm{Fe}_{25} \mathrm{Ni}_{75}$ and $\mathrm{Fe}_{75} \mathrm{Ni}_{25}$ completely random alloys are shown on figure 5 . The surrounded atom treatment of the

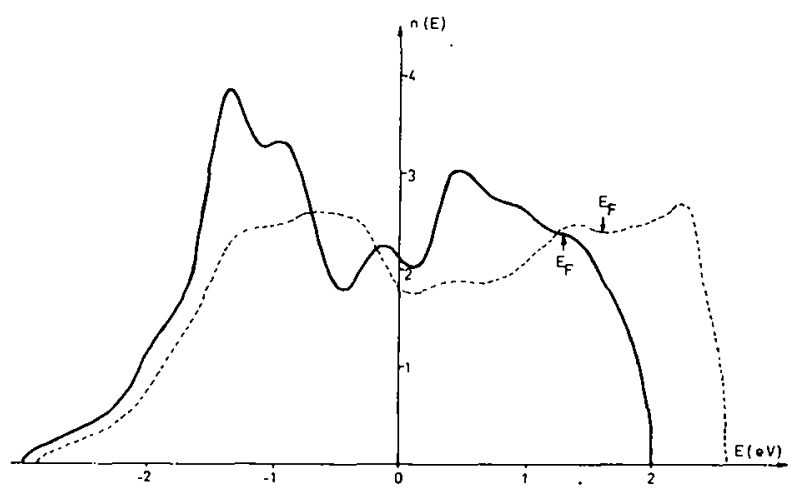

FIG. 5. - Densities of states of completely random FeNi alloys : $\left(\stackrel{\longrightarrow}{\longrightarrow} \mathrm{Fe}_{25} \mathrm{Ni}_{75}(\delta=0.76 \mathrm{eV}),(--)\right) \mathrm{Fe}_{75} \mathrm{Ni}_{25}(\delta=1.22 \mathrm{eV})$.

statistics leads to a tendency to segregation for $\mathrm{Fe}$ rich FeNi alloys. On the contrary for $\mathrm{Ni}$ rich $\mathrm{FeNi}$ alloys (around $c=0.80$ at $T=500 \mathrm{~K}$ ), the Cowley order parameter becomes negative and tendency to ordering appears. However, the exact value of the concentration and of the enthalpy of formation at this transition is quite sensitive to band parameters.

These results are then very promising. It would be therefore interesting to study also the possible influence of the effective medium, the ordering effects in the nearest neighbour shell of the central atom and to take into account charge transfer, cluster by cluster, and the s electrons.

\section{References}

[1] SOVEN, P., Phys, Rev, 156 (1967) 809.

Velicky, B., KirkPatrick, S. and Ehrenreich, H., Phys. Rev. 175 (1968) 747.

[2] Brouers. F., Cyrot. M. and Cyrot-l.ackmann, F., Phys. Rev. B 7 (1973) 4370.

Brouers. F., Ducastelle. F. Gautier. F., van der Rest. J. J. Phys. F 3 (1974) 2120.

Gautier. F., van der Rest, J. and Brouers, F., J. Phys. F 5 (1975) 1884.

[3] Falicov, L. M. and Ynourain, F., Phys. Rev. B 11 (1975) 5664

[4] Cyrot-Lackmann, F. and Cyrot, M., Solid State Commun. 22 (1977) 517.

[5] Desjongueres, M. C. and Cyrot-Lackmann, F., J. Phys. F 7 (1977) 61.
[6] Cowley, J. M., J. Appl. Phys. 21 (1950) 24.

[7] Mathieu, J. C., Durand, F. and Bonnier, E., J. Chem. Phys. 11 (1965) 1297

Hicter, P., Mathieu, J. C., Durand, F. and Bonnier, E., Ady. Phys. 16 (1967) 523.

Lupis, C. H. P. and Ellior, J. F., Act. Metall. 15 (1967) 265.

[8] Shiba. H., Prog. Theor. Phys. 46 (1971) 77.

[9] Miwa, H., Prog. Theor. Phys. 52 (1974) 1.

Kumar, V., Kumar, D. and Joshi, S. K., Phys. Rev. B 11 (1975) 2831

[10] Hultgrfen, R., Desai, P. D., Hawkins, D. T., Gleiser, M and KfLLEY, K. K., Selected values of the thermodynamic propertics of binary alloys (Am. Soc. Metals) (1973). 